\title{
Filmes de gadolínio depositados por magnetron sputtering sobre Si em diferentes potenciais de polarização
}

\author{
Gadolinium films deposited through magnetron sputtering on Si with \\ different bias
}

Francisco Alfaro $^{1,2}$, Thais Macedo Vieira ${ }^{1}$, Julio César Sagás ${ }^{1}$, Luís César Fontana ${ }^{1}$

\section{RESUMO}

Filmes finos de gadolínio (Gd) foram depositados em substratos de silício (Si) via triodo magnetron sputtering (TMS) com corrente constante de 0,5 A em atmosfera de argônio sob pressão de 0,4 Pa sem uso de aquecimento externo. Diferentes condições de deposição foram aplicadas ao substrato: potencial flutuante (1,1 V), polarização negativa $(-15,-30$ e $-50 \mathrm{~V})$, polarização positiva (5, 10 e 15 V) e substrato aterrado. Uma deposição com magnetron sputtering convencional também foi realizada em potencial flutuante $(-16 \mathrm{~V})$. As espessuras, medidas por perfilometria, foram da ordem de 0,9 $\mu \mathrm{m}$. A estrutura cristalina e a morfologia dos filmes foram estudados por difração de raios-X (DRX) com ângulo rasante e microscopia de força atômica (MFA), respectivamente. Observou-se que a polarização positiva leva a uma corrente maior comparada a aplicação de uma polarização negativa. As imagens de MFA mostram que as superfícies dos filmes têm "grãos" alongados sem diferença significativa entre as amostras de diferentes polarizações. Os espectros de DRX apresentaram as mesmas características para todos os filmes. Portanto, as diferentes polarizações não causam mudanças significativas na morfologia e cristalinidade dos filmes. Medidas das propriedades elétricas como resistividade, concentração de portadores, mobilidade eletrônica e magnetorresistência pela técnica de van der Pauw apresentaram resultados ligeiramente distintos para cada polarização.

Palavras-chave: Gadolínio; Filme fino; Pulverização catódica

\begin{abstract}
Gadolinium ( $\mathrm{Gd}$ ) thin films were deposited onto silicon substrates by triode magnetron sputtering under constant current $(0.5 \mathrm{~A})$, working gas (Ar) pressure ( 0.4 Pa) and without external heating. Different substrate biases were applied: floating potential (1.1 V), negative bias $(-15,-30$ and $-50 \mathrm{~V})$, positive bias $(5,10$ and $15 \mathrm{~V}$ ) and grounded substrate. A deposition was also made in conventional magnetron sputtering at floating potential (-16 V). The film thicknesses, measured by profilometry, were close to $0.9 \mu \mathrm{m}$. The crystalline structure and morphology of the films were analyzed by grazing incidence $\mathrm{X}$-ray diffraction (GIXRD) and atomic force microscopy (AFM). When positive bias is applied to the substrate, a higher current was measured than for negative bias. The AFM images show that the film surface has elongated "grains". No significant difference in morphology was observed for the different bias. The XRD spectra present the same characteristics for all samples. Thus, the results showed that the bias has no significant effect on the surface morphology and crystallinity of the films. Measurements of the electrical properties such as resistivity, carrier concentration, mobility electronics and magnetoresistance by the technique of van der Pauw presented slightly different results for each polarization.
\end{abstract}

Keywords: Gadolinium; Thin film; Magnetron sputtering

\footnotetext{
${ }^{1}$ Universidade do Estado de Santa Catarina - Centro de Ciências Tecnológicas - Laboratório de Plasmas, Filmes e Superfícies - Joinville (SC) - Brasil ${ }^{2}$ Centro Universitário Católica de Santa Catarina - Jaraguá do Sul (SC) - Brasil
}

Autor correspondente: Francisco Alfaro - Rua Paulo Malschitzki, 200 - Campus Universitário Prof. Avelino Marcante - Bairro Zona Industrial Norte CEP: 89.219-710 - Joinville - SC - Brasil

Email: francisco@alfaro.com.br

Recebido: 24/08/2016 Aprovado: 30/08/2016 


\section{INTRODUÇÃO}

O efeito magnetocalórico (EMC) atrai atenção no ambiente acadêmico e industrial devido a aplicações relacionadas à eficiência energética e ao desenvolvimento de refrigeradores magnéticos domésticos ${ }^{(1)}$. Os dispositivos eletrônicos de micro e nano escala modernos requerem técnicas de refrigeração em micro-escala, portanto, tem-se desenvolvido pesquisas investigando o EMC em filmes finos ${ }^{(2,3,4)}$

O efeito magnetocalórico de uma substância é caracterizado pela variação adiabática da temperatura e pela variação isotérmica da entropia magnética quando o material está sob a influência de um campo magnético variável. Dados experimentais indicam que as características que descrevem o EMC são maiores em transições de fase magnéticas ${ }^{(5)}$. Para se entender a origem do efeito magnetocalórico utiliza-se atualmente aproximações termodinâmicas, pois as considerações microscópicas não estão suficientemente desenvolvidas ${ }^{(6)}$.

Uma das técnicas que é amplamente usada, dentro da deposição física a vapor (physical vapor deposition - PVD) é a técnica de pulverização catódica (magnetron sputtering), que é baseada em um plasma, no qual os átomos de um gás inerte são ionizados e acelerados por uma diferença de potencial entre o cátodo e ânodo. O filme é o resultado da interação que ocorre entre os átomos ionizados e a superfície do alvo. A interação provoca a ejeção dos átomos que são condensados no substrato, formando assim o filme. Essa condensação e a criação do filme ocorrem fora do equilíbrio termodinâmico ${ }^{(7)}$, portanto, um bom controle das condições termodinâmicas e cinéticas implicam diretamente na relação das propriedades estruturais, ópticas, elétricas e mecânicas do filme. Além disso, vários estudos mostram que o fluxo de energia, o ângulo de incidência e a natureza do bombardeamento são importantes para as propriedades do filme depositado ${ }^{(8-12)}$.

Portanto, neste trabalho, filmes finos de Gd foram depositados em um sistema tríodo magnetron sputtering (TMS) sob diferentes condições de polarização, com o intuito de verificar se tais mudanças na transferência de energia para o filme afetam sua estrutura e morfologia.

\section{MATERIAIS E MÉTODOS}

Os substratos foram preparados a partir de fraturas de uma lâmina de Si (111) tipo $\mathrm{p}$, das quais se utilizaram pedaços que mantiveram uma área superficial entre 1 e $2 \mathrm{~cm}^{2}$ e que foram submetidos ao seguinte processo de limpeza: 5 minutos em água deionizada $\left(\mathrm{H}_{2} \mathrm{O} \mathrm{DI}\right)$ corrente, 10 minutos em ácido sulfúrico $\mathrm{H}_{2} \mathrm{O}_{2}+4 \mathrm{H}_{2} \mathrm{SO}_{4}, 5$ minutos em $\mathrm{H}_{2} 0$ DI corrente e 1 minuto em uma solução aquosa de ácido fluorídrico ( $\left.\mathrm{HF}+20 \mathrm{H}_{2} \mathrm{O} \mathrm{DI}\right)$.

O reator de deposição consiste de uma câmara de aço inoxidável. O sistema de vácuo é composto por uma bomba mecânica e uma bomba turbomolecular, permitindo atingir pressões da ordem de $10^{-3} \mathrm{~Pa}$. O alvo de $\mathrm{Gd}$ tem $99,5 \%$ de pureza conforme informações do fornecedor (Testbourne Ltd) e $100 \mathrm{~mm}$ de diâmetro. As deposições foram realizadas à temperatura ambiente sem sistema de aquecimento ou de refrigeração do substrato. A atmosfera era de argônio sob pressão de 0,4 Pa aproximadamente, medida por um sensor capacitivo. Cada deposição durou da ordem de 16 minutos, que foi o tempo estimado para produzir filmes da ordem de $0,9 \mu \mathrm{m}$, sob corrente constante de $0,5 \mathrm{~A}$ e potência aproximada de $180 \mathrm{~W}$ no cátodo. A distância alvo-substrato foi fixa em $7,3 \mathrm{~cm}$

As amostras foram diferenciadas conforme a polarização aplicada ao substrato durante a deposição. As polarizações aplicadas foram $+5 \mathrm{~V},+10 \mathrm{~V},+15 \mathrm{~V},-15 \mathrm{~V},-30 \mathrm{~V},-50 \mathrm{~V}$, potencial flutuante de $1,1 \mathrm{~V}$ e substrato aterrado $(0 \mathrm{~V})$. Todas as polarizações foram feitas no modo de corrente contínua. Para cada amostra foi feita uma réplica, ou seja, outra deposição nas mesmas condições. Todas as condições de polarização foram aplicadas com uso de uma tela distante $2 \mathrm{~cm}$ do alvo. A tela é mantida aterrada, sendo, portanto, o anodo da descarga. A tela possui uma malha de $1 \times 1 \mathrm{~mm}$ e é feita de aço inoxidável. Apenas uma condição foi realizada sem uso da tela, que foi a de potencial flutuante, na qual se alterou o valor da tensão de $1,1 \mathrm{~V}$ para $-16 \mathrm{~V}$.

Pequenos retângulos de $\mathrm{Si}\left(\sim 2 \times 10 \mathrm{~mm}^{2}\right)$ foram utilizados como máscara para medir as espessuras, feitas por perfilometria. Para determinar a espessura foram realizadas cinco medidas em cada amostra.

Os difratogramas de raios-X foram obtidos com ângulo de incidência rasante fixado em $1^{\circ}$ e o detector movendo-se e fazendo a varredura $2 \theta$ na velocidade de $1 \%$ min. O aparelho utilizado é da marca Shimadzu modelo DRX-7000 com alvo de cobre de emissão predominante no comprimento de onda $\mathrm{K}_{\alpha}=1,5406 \AA$, $30 \mathrm{~mA}$ de corrente e $40 \mathrm{kV}$ de diferença de potencial.

As imagens de microscopia de força atômica foram obtidas com o aparelho Nanosurf modelo Nanite B operando no modo estático com uma ponta não magnética de Si com raio de $\sim 20 \mathrm{~nm}$.

Medidas de resistividade e magnetorresistência foram realizadas em um aparelho de medição por efeito Hall, marca ECOPIA modelo HMS 5500, utilizando o método de van der Pauw. As amostras foram submetidas a uma variação de temperatura de $100 \mathrm{~K}$ a $300 \mathrm{~K}$, obtendo-se medidas de resistividade, concentração de portadores, mobilidade e magnetorresistência de 10 em $10 \mathrm{~K}$ para um campo magnético de $0,53 \mathrm{~T}$.

\section{RESULTADOS E DISCUSSÃO}

\section{Curvas corrente-tensão do substrato}

As relações entre tensão e corrente aplicadas ao substrato são mostradas na Figura 1. Para tensões positivas a corrente atinge valores de cerca de $-200 \mathrm{~mA}$ para um potencial de $24 \mathrm{~V}$, enquanto que para tensões entre $0 \mathrm{e}-70 \mathrm{~V}$ a corrente varia entre 4,6 e 5,8 mA. No caso da polarização positiva do substrato, existe uma corrente predominantemente eletrônica, enquanto para a polarização negativa tem-se um fluxo líquido de cargas positivas 
para o substrato. Como o plasma é confinado entre o alvo e a tela, a região entre a tela e o substrato pode ser considerada uma região de pós-descarga, com um baixo campo elétrico devido à diferença de potencial entre a tela e o substrato. Os maiores valores de corrente observados para uma polarização positiva podem ser atribuídos à maior mobilidade eletrônica nestas condições.

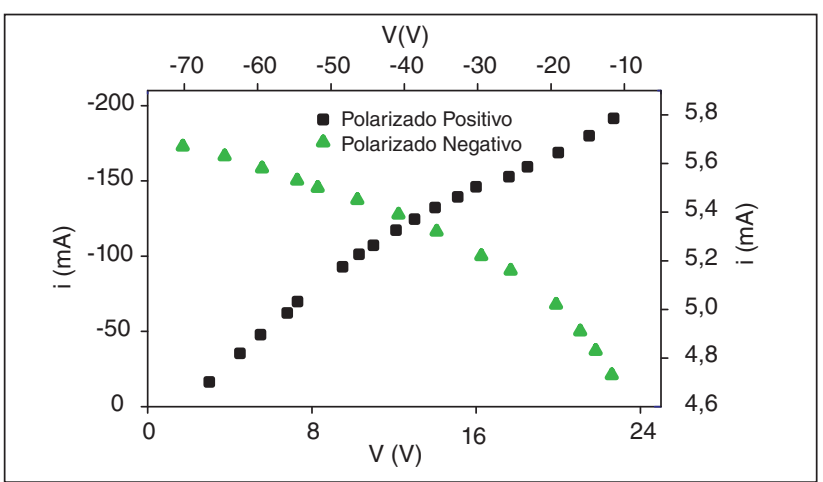

Figura 1: Comportamento da corrente no substrato em função da tensão aplicada.

\section{Espessura dos filmes}

A Figura 2 apresenta um exemplo de uma medida de perfilometria para o filme depositado a $+10 \mathrm{~V}$. Nota-se uma variação na altura do perfil de aproximadamente $0,9 \mu \mathrm{m}$, entre as regiões com e sem filme. As médias das espessuras para cada amostra estão mostradas na Figura 3. Percebe-se que as espessuras variaram entre 0,6 e 1,1 $\mu \mathrm{m}$ para as amostras com tela. A espessura do filme depositado por magnetron sputtering convencional pode atingir até $1,5 \mu \mathrm{m}$, uma vez que a taxa de deposição é maior devido à ausência da tela, onde parte do material é depositado no TMS

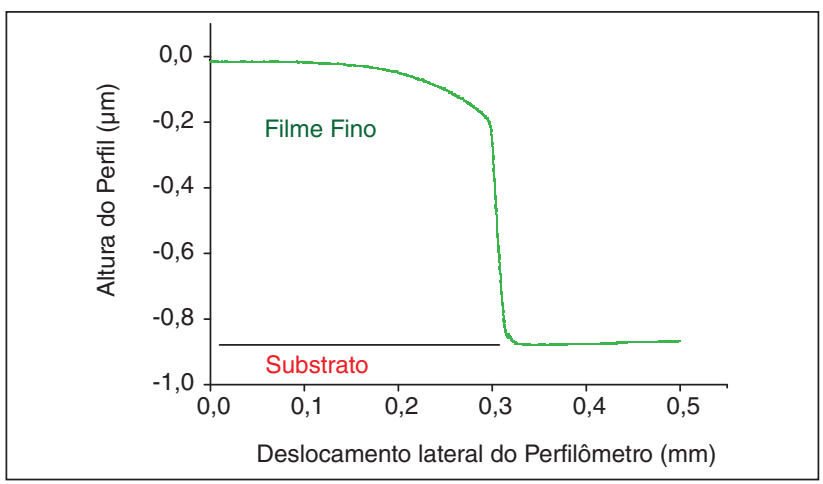

Figura 2: Perfilometria da amostra submetida a polarização positiva de $10 \mathrm{~V}$.

\section{Difração de Raios-X}

A Figura 4 mostra os difratogramas de raios- $\mathrm{X}$ para as amostras polarizadas em $+15 \mathrm{~V},-50 \mathrm{~V}$, aterrado $(0 \mathrm{~V})$, potencial flutuante com e sem tela $(1,1 \mathrm{~V}$ e $-16 \mathrm{~V}$, respectivamente). Nenhuma mudança significativa foi percebida entre os difratogramas e todos coincidiram com os picos esperados para o Gd puro.

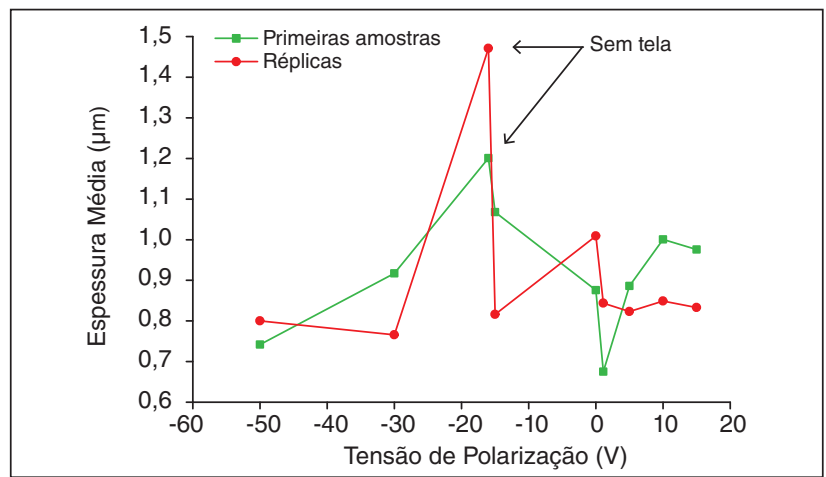

Figura 3: Média das espessuras das amostras.

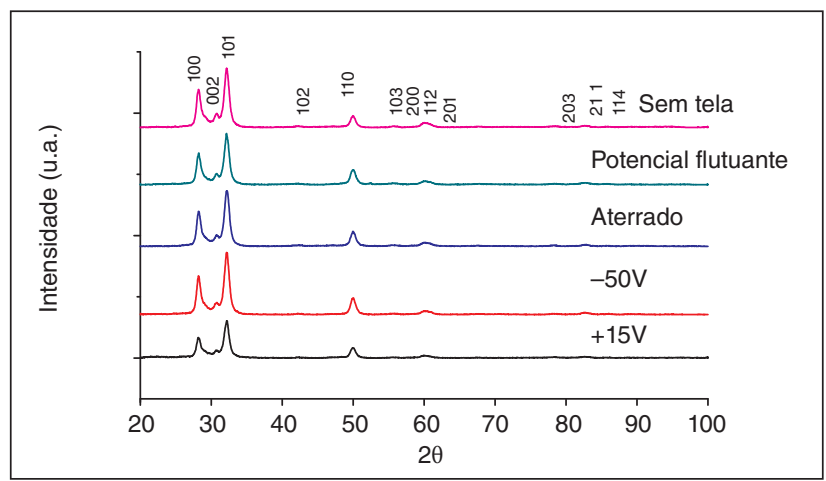

Figura 4: Difração de raios- $X$ rasante das amostras polarizadas em $+15 \mathrm{~V},-50 \mathrm{~V}$, aterrado $(0 \mathrm{~V})$, potencial flutuante com e sem tela $(+1,1 \mathrm{~V}$ e $-16 \mathrm{~V}$, respectivamente).

\section{Microscopia de Força Atômica}

A técnica de microscopia de força atômica foi utilizada para todas as amostras, varrendo áreas de $10 \times 10 \mathrm{~mm}^{2}$ e $5 \times 5 \mathrm{~mm}^{2}$. Todas as imagens apresentaram "grãos" alongados na superfície varrida pelo microscópio. A Figura 5 mostra a imagem de MFA para a amostra polarizada em $+15 \mathrm{~V}$.

O tratamento via software das imagens de MFA permitiu analisar as rugosidades rms $\left(\mathrm{R}_{\mathrm{q}}\right)$ e média $\left(\mathrm{R}_{\mathrm{a}}\right)$ de cada uma das amostras. Esses resultados estão na Figura 6. As medidas de rugosidade não apresentaram variações significativas entre as amostras.

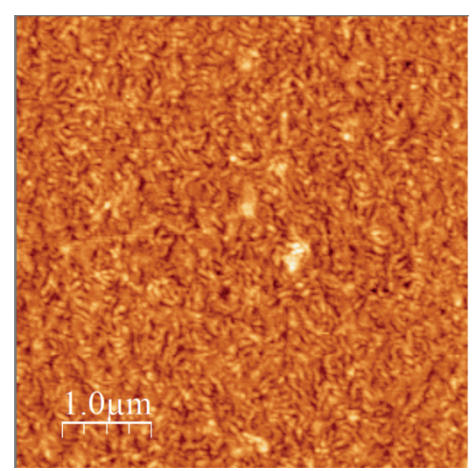

Figura 5: Imagem de microscopia de força atômica realizada na amostra polarizada a $+15 \mathrm{~V}$. Rugosidade média de $2,57 \mathrm{~nm}$. 


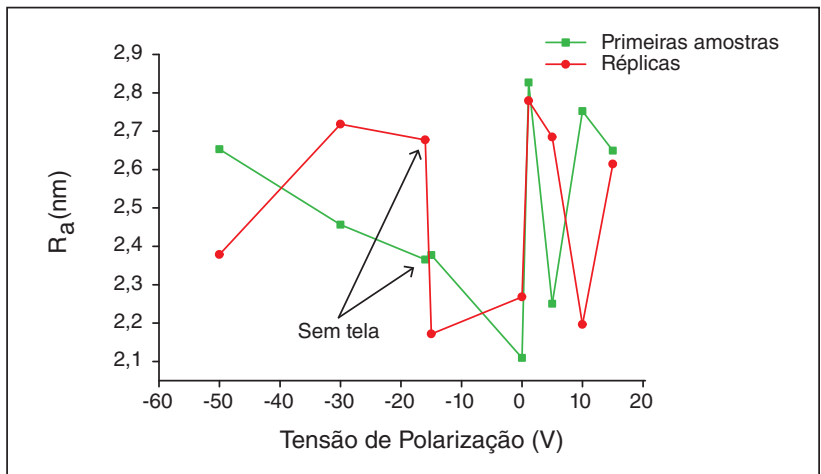

Figura 6: Rugosidade média das amostras.

\section{Resistividade e Magnetorresistência}

As medidas de resistividade em função da temperatura de algumas amostras são mostradas na Figura 7. Em todos os casos, a resistividade aumenta com a temperatura, como é típico para os metais. Nota-se que existe uma pequena diferença no valor da resistividade conforme a polarização da amostra e o não uso da tela. Essa diferença não está relacionada à espessura do filme, pois não existe um padrão entre os dois parâmetros. Esse comportamento da resistividade em função da polarização das amostras indica que as diferentes condições de deposição afetam as propriedades elétricas, como será discutido a seguir.

A Figura 8 mostra as medidas de magnetorresistência em função da temperatura para amostras polarizadas em $+15 \mathrm{~V}$, $-50 \mathrm{~V}$, aterrado, potencial flutuante com e sem tela. Todas as amostras apresentaram a mesma tendência de aumento da magnetorresistência com a temperatura e uma queda "brusca" em torno de $270 \mathrm{~K}$, sendo que a temperatura de Curie do Gd é em torno de $290 \mathrm{~K}$.

A concentração de portadores e a mobilidade eletrônica apresentam comportamento similar ao da magnetorresistência, como pode ser visto nas Figuras 9 e 10, respectivamente. Notase que a mobilidade eletrônica é similar para as diferentes amostras, mas a concentração de portadores, especialmente em baixas temperaturas, é dependente da polarização. Logo, o comportamento da resistividade se deve ao comportamento do

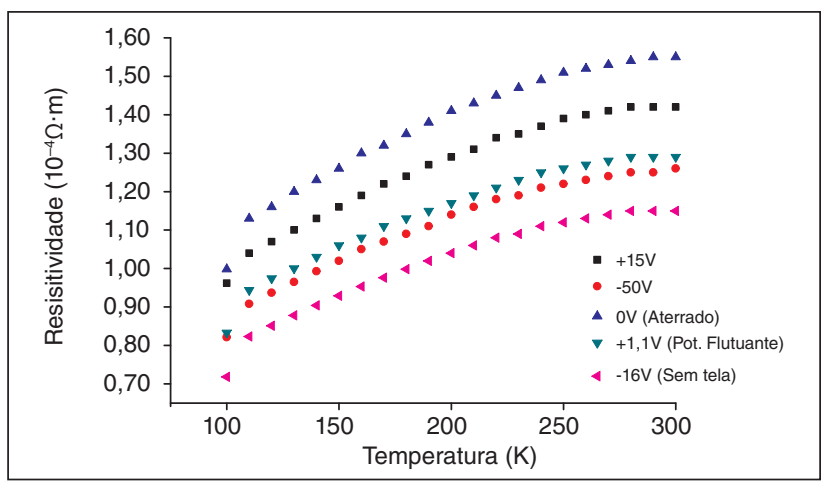

Figura 7: Medidas de resistividade em função da temperatura para amostras polarizadas em $+15 \mathrm{~V},-50 \mathrm{~V}$, aterrado $(0 \mathrm{~V})$, potencial flutuante com e sem tela $(+1,1 \mathrm{~V}$ e $-16 \mathrm{~V}$, respectivamente).

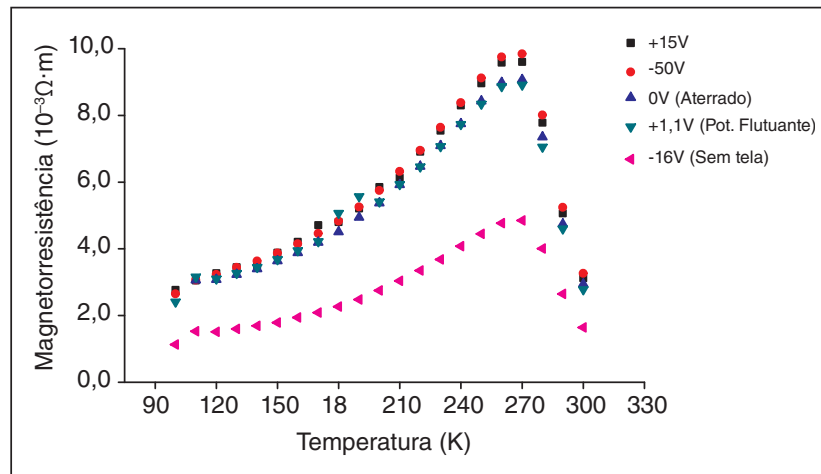

Figura 8: Medidas de magnetorresistência em função da temperatura para amostras polarizadas em $+15 \mathrm{~V},-50 \mathrm{~V}$, aterrado $(0 \mathrm{~V})$, potencial flutuante com e sem tela $(+1,1 \mathrm{~V}$ e $-16 \mathrm{~V}$, respectivamente).

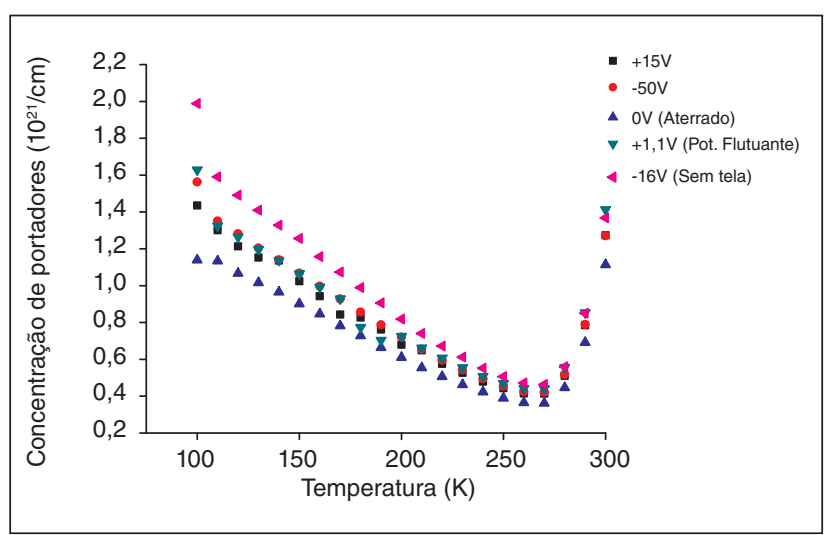

Figura 9: Medidas de concentração de portadores em função da temperatura para amostras polarizadas em $+15 \mathrm{~V},-50 \mathrm{~V}$, aterrado $(0 \mathrm{~V})$, potencial flutuante com e sem tela $(+1,1 \mathrm{~V}$ e $-16 \mathrm{~V}$ respectivamente).

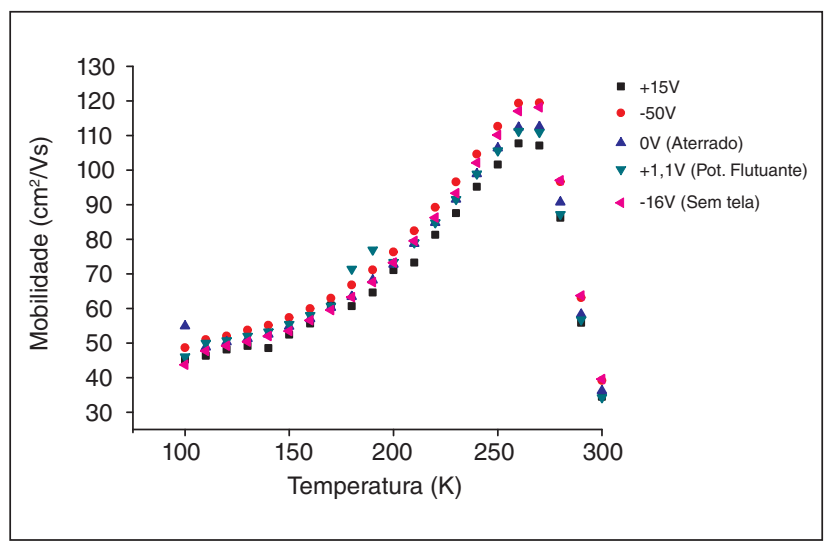

Figura 10: Medidas de mobilidade eletrônica em função da temperatura para amostras polarizadas em $+15 \mathrm{~V},-50 \mathrm{~V}$, aterrado $(0 \mathrm{~V})$, potencial flutuante com e sem tela $(+1,1 \mathrm{~V}-16 \mathrm{~V}$, respectivamente).

número de portadores. Para o filme depositado em condição aterrada, a concentração de portadores é menor, implicando em uma maior resistividade, enquanto para o filme depositado por magnetron sputtering convencional, verifica-se uma maior densidade de portadores, resultando em uma menor resistividade. 
O comportamento da magnetorresistência com a polarização, em particular, para o caso sem tela ainda precisa ser melhor estudado.

Nota-se ainda que existe um ponto crítico no comportamento da magnetorresistência, concentração de portadores e mobilidade eletrônica em relação a temperatura. De 100 até 270 $\mathrm{K}$ diminui a concentração de portadores e aumenta a mobilidade eletrônica conforme o acréscimo de temperatura, enquanto que no intervalo de $270 \mathrm{~K}$ a $300 \mathrm{~K}$ o comportamento se inverte. Isso indica que a transição de fase magnética do Gd interfere na condução elétrica.

\section{CONCLUSÃO}

Filmes de Gd foram depositados em um sistema TMS sobre Si (111) em diferentes condições de polarização. Embora se observe uma maior corrente para polarizações positivas (bombardeamento predominantemente eletrônico), as análises por microscopia de força atômica e difração de raios-X não mostraram nenhuma diferença significativa na estrutura e morfologia dos filmes. No entanto, a análise das propriedades elétricas pela técnica de van der Pauw mostra que a resistividade depende da polarização: o bombardeamento iônico do filme, durante a deposição, contribui para diminuir resistividade e a magnetoresistência. Essa diminuição da resistividade está associada ao aumento na concentração dos portadores de carga no filme.

\section{AGRADECIMENTOS}

À PUCPR por permitir a utilização do equipamento DRX e ao CEITEC pela disponibilização das lâminas de silício. Essa pesquisa foi financiada pelo projeto UNIVERSAL/CNPq 455896/2014-3.

\section{REFERÊNCIAS}

1. TISHIN, A.M.; SPICHKIN Y.I., Series in Condensed Matter Physics: The Magnetocaloric Effect and its Applications, Institute of Physics, London, 2003, 3p.

2. AKKERA, H.S.; CHOUDHARY, N.; KAUR, D., Martensitic phase transformations and magnetocaloric effect in $\mathrm{Al}$ co-sputtered Ni-Mn-Sb alloy thin films. Mater. Sci. Eng. B. 198, 2015, pp. 113-119.

3. KIM, M.; JUNG, M.; KIM, C.M.; LIM, S.H., Effects of Ar pressure on magnetic and magnetocaloric properties of sputtered Er-Co thin films. Phys. B, 476, 2015, pp. 175-178.

4. GADIOLI G.Z.; ROUXINOL, F.P.; GELAMO, R.V.; SANTOS A.O.; CARDOSO, L.P.; MORAES, M.A.B., Magnetism in Gd-W films. J. Appl. Phys. 103, 2008, 096916p.

5. PECHARSKY, V.K.; GSCHNEIDNER Jr., K.A.; PECHARSKY, V.K.; TISHIN, A.M; Thermodynamics of the magnetocaloric effect. Phys. Rev. B. 64, 1997, 144406p.

6. TISHIN, A.M.; SPICHKIN, Y.I., Recent progress in magnetocaloric effect: Mechanisms and potential applications. International Journal of Refrigeration, v. 37, 2014, pp. 223-229.

7. OHRING, M., The Material Science of Thin Films, Academic Press, San Diego, 1992.

8. AUCIELLO, O. KELLY, R. Ion Bombardment Modification of Surfaces: Fundamentals and Applications, Elsevier Science Ltd, Amsterdam, 1984.

9. KERSTEN, $\mathrm{H}$. et al. The energy balance at substrate surfaces during plasma processing. Vacuum, v. 63, 2001, pp. 385-431.

10. MAHIEU, S. et al. Biaxial alignment in sputter deposited films. Thin Solid Films, v. 515, 2006, pp.1229-1249.

11. BORNHOLDT, S. et al. Characterization of the energy flux toward the substrate during magnetron sputter deposition of $\mathrm{ZnO}$ thin films. Plasmas Sources Sci. Technol., v. 22, 025019, 2013, 8p.

12. ANDERS, A. A structure zone diagram including plasma-based deposition and ion etching. Thin Solid Films, v. 518, 2010, p. 4087-4090. 